\title{
Editorials
}

\section{The role of psychopharmacology in suicide prevention}

\author{
KATE E. A. SAUNDERS and KEITH HAWTON
}

\begin{abstract}
The potential role of psychopharmacology in suicide prevention is often minimised. This may to some extent reflect that few medication trials have specifically focussed on prevention of suicidal behaviour - indeed this outcome is often not reported in trials. However, there is reasonably strong evidence that lithium may reduce risk of suicide, the postulated mechanism being a specific effect on aggression. Evidence is lacking with regard to any protective effect of other mood stabilizers. Clozapine may reduce suicidal behaviour in patients with schizophrenia, with reduction of affective symptoms being a possible explanation. The role of antidepressants in relation to suicide risk is highly controversial, especially in children and adolescents. It is unclear whether minor tranquillizers or hypnotics can assist in suicide prevention, although they can reduce the anxiety symptoms that may occur during initial treatment with SSRI antidepressants. It is also uncertain whether psychopharmacology has a role in preventing suicidal behaviour in people with personality disorders. Despite the limitations of the evidence we contend that suicide risk should be an important factor in deciding when and what to prescribe.
\end{abstract}

Declaration of Interest: We have no interests to declare.

The role of psychotropic medication in suicide prevention has been downplayed despite evidence that some agents reduce suicidal behaviour. Medication plays a relatively minor role in most suicide prevention strategies. Recently there have been concerns that some of our most commonly prescribed medications may be associated with increased risk of suicide. Given that approximately $90 \%$ of those who die by suicide and a similar proportion of those who attempt suicide have psychiatric disorders (Cavanagh et al., 2003; Haw et al., 2001) the need to utilise pharmacological means to reduce suicide is clearly important.

\section{LITHIUM}

There is good evidence that lithium reduces risk of relapse in patients with mood disorders (Geddes et al., 2004) and there have been many claims that lithium also

Address for correspondence: Professor K. Hawton, Centre for Suicide Research, University Department of Psychiatry, Warneford Hospital, Oxford OX3 7JX (United Kingdom).

Fax: +44-1865-793101

E-mail: keith.hawton@psych.ox.ac.uk reduces risk of suicide (Baldessarini et al., 2003). Most investigations have been observational studies. For example, in one such study based on data collected by a health maintenance organisation in the USA, a 2.7 times greater risk of suicide was found for patients prescribed divalproex (valproate) compared to those prescribed lithium (Goodwin et al., 2003). However, such studies, especially those based on lithium clinic samples, are likely to produce biased findings. Any beneficial effect might reflect compliance with medication being greater in patients attending a clinic. There may also be selective prescribing to patients at risk. Furthermore, deaths by other causes are often not considered. Given the potential physical side-effects of lithium on thyroid and renal function it is possible that a benefit of lithium in preventing suicide might be offset by increased risk of death from physical disorders.

A systematic review and meta-analysis of evidence on possible anti-suicidal effects of lithium from randomised controlled trials (RCTs) lasting at least three months in patients with mood disorders, in which there was comparison of lithium versus placebo, or versus any active drugs, was conducted by Cipriani et al. (2005). 32 RCTs met the inclusion criteria. These included 1,377 patients randomised to lithium, and 2,052 to other compounds. 19

Epidemiologia e Psichiatria Sociale, 18, 3, 2009 
trials compared lithium with placebo, nine with carbamazepine, three with lamotrigine and one with divalproex. The other trials were comparisons with antidepressants, namely imipramine, amitriptyline, maprotiline and mianserin. Multiple comparisons were included in some trials.

Seven trials reported suicide as an outcome and an event occurred in at least one arm of the trial. In these trials 2 out of 503 people prescribed lithium died by suicide compared with 11 out of 601 patients prescribed placebo or comparator drugs (OR 0.26, 95\%CI 0.09-0.77). Thus there was evidence of a significant reduction of suicide in those prescribed lithium (although this finding was associated with significant heterogeneity). Surprisingly few trials included data on deliberate self-harm/attempted suicide. Therefore, results for these outcomes were combined with those for suicide. There was a lowered risk of fatal or non-fatal suicidal behaviour, this occurring in just 6 out of 670 patients in the lithium treated groups compared with 18 out of 781 in the groups of patients receiving comparator drugs (OR 0.21, 95\%CI 0.08-0.50), although this result was also associated with significant heterogeneity. Finally, deaths from all causes were examined. There was a much reduced overall risk of death from any cause in patients treated with lithium than in those in the comparison groups (9/696 vs. 22/788, OR 0.42 , 95\% CI 0.21-0.87), although this result was again associated with significant heterogeneity. The apparent beneficial effect of lithium on suicide risk was not offset by an increase in deaths from other causes. In post-hoc analyses there was no significant difference for the above outcomes between the results of trials that compared lithium with placebo and those that compared lithium with active drugs. While the effects of lithium on suicidal behaviour were less in this meta-analysis of RCTs than reported in non-randomised studies, lithium appeared to reduce the risk of suicide by approximately $60 \%$. The reduction for suicide and deliberate self-harm/attempted suicide combined was approximately $70 \%$. Overall, the reduction in deaths from all causes was of the order of $60 \%$. A recent randomised controlled trial of lithium versus placebo survival analysis revealed no difference of suicidal acts between lithium and placebo treated depressed individuals (Lauterbach et al., 2008). However, post hoc analysis revealed that all of the completed suicides occurred in the placebo group. There were a high number of drop-outs and the total number of suicidal events was just 17 such that these results should be interpreted with caution, but the study does appear to support a role for lithium in protecting those with affective disorders against suicidal acts with a fatal outcome.
Currently, therefore, in spite of the drawbacks of lithium therapy, it appears it may be the treatment of choice for people with BPD who are at risk of suicide, and may also have role in protecting those with depressive disorders against fatal suicidal acts.

The mechanism by which lithium confers this protective effect is unclear. It may relate to its mood stabilising properties but the effect on suicidal behaviour has been observed even when mood stabilisation has not occurred (Muller-Oerlinghausen et al., 1992). The anti-aggressive properties of lithium (Wickham \& Reed, 1987) are likely to be relevant as aggression is associated with suicidal behaviour (Angst \& Clayton, 1986).

\section{ANTICONVULSANTS}

While the protective effect of lithium is reasonably well established the role of other mood stabilisers in preventing suicide has received far less attention. Given the recent trend towards the use of anticonvulsant medications in mood stabilisation their role in suicide prevention is of increasing relevance in the treatment of bipolar disorder. Some observational studies suggest that the suicide risk is higher in those patients taking sodium valproate than those on lithium (Goodwin et al., 2003), but drawing firm conclusions is difficult not least because of the possible confounding by indication i.e. the reason for the individual receiving valproate is related to a more severe form of the illness or one associated with higher rates of suicide, for example rapid cycling (Hawton et al., 2005b). Such observations may also relate to the increased risk of suicide associated with stopping lithium (Tondo \& Baldessarini, 2000). Some authors have reported no difference in suicide rates between those taking lithium and those on other mood stabilisation (Yerevanian et al., 2003). The rare nature of suicide can make the interpretation of such data difficult. Smith et al. (2009) have recently published a large observational study of Danish healthcare records in which they have captured up to 30 times more suicides than earlier studies. They examined prescription collections for all patients prescribed anticonvulsants or lithium who had also received a prescription for an antipsychotic (this was done to exclude all those taking anticonvulsants for non psychiatric reasons). They found that consistent purchase of anticonvulsants was associated with a reduction in suicide when compared with those that had only picked up one script. The magnitude of the reduction was similar to the reduction in suicides seen when comparing those who consistently purchased lithium versus those who did not. Comparison

Epidemiologia e Psichiatria Sociale, 18, 3, 2009 
between the groups was more difficult because of confounding indicators. Also, the method employed to select cases excluded all those with bipolar illness who had not required antipsychotic medication (i.e. those who had less severe forms of the illness) and may have included individuals taking anticonvulsants for epilepsy but who had at some point required antipsychotic medication. There were lower rates of suicide than reported in the retrospective cohort study performed by Goodwin et al. (2003). $65 \%$ of those on anticonvulsants were prescribed carbamazapine so that any protective effect may have been specific to this medication rather than a class effect. In an earlier study of use of anticonvulsants and lithium in an inpatient sample Sondergard et al. (2008) found that while anticonvulsants reduced suicide risk, this risk decreased even further on switching to lithium.

Yerevenian et al. found additional prescribing of antidepressants (Yerevanian et al., 2007b) or antipsychotics (Yerevanian et al., 2007a) in addition to mood stabilisers was associated with increases in suicide. Furthermore, those taking antipsychotic monotherapy or antidepressant monotherapy were at highest risk of suicide. The addition of a mood stabiliser reduced this to an intermediate risk with the lowest suicide rate being among those that were on mood stabilisation alone. These results should be interpreted cautiously as it was a retrospective, non-randomised study and the groups prescribed medications in addition to mood stabilisers were likely to be more unwell and therefore at higher risk.

\section{ANTIPSYCHOTICS}

Suicidal acts are common in those suffering from psychotic illnesses. Approximately 5\% of individuals will die by suicide (Palmer et al., 2005) and attempted suicide is common (Fenton et al., 1997). Clozapine has been identified in some studies as having a protective effect. Meltzer \& Okayli (1995) demonstrated a reduction in suicide attempts from 5 to 0 in patients with treatment resistant schizophrenia/schizoaffective disorder switched to clozapine. They also noted no difference in baseline suicidal ideation between the treatment resistant group and the treatment responsive group and thus concluded that clozapine was superior than the first generation antipsychotics (FGAs) taken by the rest of the participants. However, because the authors did not report follow-up data for those in the treatment responsive group, or the treatment resistant group who did not commence clozapine, only limited conclusions can be drawn. Walker et al. (1997) conducted a retrospective study of 67,072 patients registered on the Clozaril National Register and found that current users of clozapine had a lower mortality rate than recent or past users. There was no control group, however, and including past users as a comparison group is likely to have introduced bias given that medication discontinuation is associated with poorer clinical outcome (Roy, 1982). Sernyak et al. (2001) studied a large veteran sample and compared those on clozapine with a matched group taking FGAs. The group receiving clozapine were significantly less likely to die during the study period (3 years post discharge) than the control group but this reduction in mortality was entirely attributable to a lower rate of respiratory disorders in the clozapine group. No difference in suicide or accidental death was observed.

The Intersept (International Suicide Prevention Trial) trial was established to compare clozapine and olanzapine in the treatment of individuals with schizophrenia or schizoaffective disorder who were deemed to be at high risk of suicide (Meltzer et al., 2003). It was a randomised, open label trial which took place across a number of major centres internationally. Olanzapine was chosen because of suggestions that it reduced suicide and suicidal ideation when compared with haloperidol (Tran et al., 1997). At 2 year follow-up clozapine was associated with a significant reduction in suicidality, suicide attempts and fewer interventions to prevent suicide. There were also fewer deaths by suicide but this was not statistically significant. This study requires replication.

The mechanism of any protective effect of clozapine against suicidal behaviour is unclear. It may relate to its effect on depressive symptoms as suggested by the lower use of antidepressants and lower mood symptom scores in patients on clozapine in Intersept (Meltzer et al., 2003). This postulated mechanism is in keeping with the elevated risk of suicide associated with affective symptoms in schizophrenia (Hawton et al., 2005a). It has been proposed that suicidal behaviour is a symptom domain independent of depression or anxiety (Lindenmayer et al., 2003) and thus clozapine may have a unique mechanism of action in this domain. Superior efficacy with regard to treatment resistant psychotic symptoms was not found in Intersept, and both patient groups were seen with the same frequency such that levels of contact also does not account for the effect of clozapine on suicidal behaviour.

Khan et al. (2001) studied over 10,000 patients registered on the FDA database to compare suicide risk in those taking placebo vs first generation vs second generation antipsychotics. Despite the large sample size no significant difference in suicidal behaviour was found between the groups. This may simply reflect the sample characteristics of those entering placebo control trials.

Epidemiologia e Psichiatria Sociale, 18, 3, 2009 
FGAs appear not to have any demonstrable effect in reducing suicidal behaviour (Winokur \& Tsuang, 1975). Their side effects have been suggested as a risk factor for suicide but there is limited evidence to support this. The role of second generation antipsychotics other than clozapine in suicide prevention has not been systematically explored further than the observation that consistent medication compliance is associated with a decreased risk of death by suicide (Ward et al., 2006).

\section{ANTIDEPRESSANTS}

The role of antidepressant treatment in suicide prevention remains a controversial issue, with many authors reporting reductions in suicide (Baldessarini et al., 2006; Moller, 2006; Rihmer \& Akiskal, 2006) but meta analyses of short-term treatment finding evidence of increases in suicide (Fergusson et al., 2005; Khan et al., 2003). Higher rates of prescription of antidepressants have been found to correlate with lower suicide rates in Hungary, Sweden, Australia and the USA, but this has not been demonstrated in other countries such as Iceland, Japan or Italy (Mann et al., 2005). It has been suggested that this may relate to other factors such as lower alcohol use and generally lower baseline rates of suicide.

Concerns regarding the safety of antidepressants in children and adolescents were first raised in the UK by the Medicines and Healthcare products Regulatory Agency (MHRA) in 2003. This was followed in March 2004 by a warning released by the US Food and Drugs Administration (FDA) that there was an increased risk of suicide with the 10 newer antidepressants. Much of the concern has focussed on the prescribing of such medications to younger age groups but recent meta-analysis has concluded that the benefits of antidepressant treatment appear to be greater than the risk posed by the emergence of or increases in suicidal ideation across all indications (Bridge et al., 2007). Suicide is the third leading cause of death in young people and $90 \%$ of youth suicides are in those whose depressive symptoms have remained untreated at the time of their deaths (Leon et al., 2004). In patient populations lower rates of suicide are observed in those treated with antidepressants and adolescents who receive 6 months of treatment have lower rates of suicide when compared with those who receive just 2 months (Valuck et al., 2004). The risk had been judged to be highest in the initial phase of antidepressant treatment but Simon et al. (2006) found that the risk of death in children and adults was not increased in the first month of treatment compared with the following months. The risk of suicide attempt was in fact highest in the month before starting antidepressant treatment and declined progressively after starting medication.

The safety warnings had a major impact on prescribing behaviour. Rather than reducing suicide rates, Gibbons et al. (2007) reported that the reduction in prescribing of antidepressants that followed the warning was associated with an increase in suicide in children and adolescents in the USA and the Netherlands. This finding was not replicated in the UK in an ecological study which found no change in suicide or non-fatal self-harm in under-18s over the first 3 years of reduced prescribing (Wheeler et al., 2008).

The role of antidepressants in suicide prevention in bipolar disorder is also contentious. Bipolar disorder is dominated by depression and for many years there was the assumption that this was best treated as if it were unipolar depression, and many patients are treated on this basis until their bipolarity becomes more evident. It is likely that any trial of patients with unipolar depression will in fact include some individuals with bipolar disorder who are yet to have experienced a manic/hypomanic episode. Patients with unrecognised bipolar disorder (therefore those likely to receive antidepressant treatment) have higher rates of attempted suicide than those who are recognised as having bipolar disorder or those who do not have it (Shi et al., 2004). This may have considerable impact on the outcomes reported in antidepressant trials given the previously mentioned difficulties in identifying such patients. Several authors have suggested that antidepressant treatment in this group increases the prevalence of suicidal ideation and behaviour (Goldberg et al., 2005; Yerevanian et al., 2007b). However, a prospective study from the STEP-BD trial did not replicate these results. They found no association was found between new onset suicidality and increased antidepressant exposure or initiation of antidepressant treatment (Bauer et al., 2006).

\section{MINOR TRANQUILLIZERS}

The possible role of minor tranquillizers, including hypnotics, in suicide prevention has been linked to earlier observations that anxiety and panic attacks may represent an early warning sign for suicide in those who are depressed (Fawcett et al., 1990). In more recent years tranquillizers and hypnotics have also been used to lessen the increase in anxiety experienced by some patients commencing treatment with SSRIs, a time that has been linked to increases in suicidal ideation. Furthermore, some have 
suggested that suicide could be reduced by aggressively treating with benzodiazepines (Fawcett et al., 1990). Studies dating back as far as the 1960s have reported the development of suicidal thoughts and tendencies following treatment with diazepam (Hall \& Joffe, 1972; Ryan et al., 1968). In depressed patients benzodiazepines have been found to worsen suicidal ideation (Jonas \& Hearron, 1996) and those who use benzodiazepines are more likely to die by suicide (especially if they are young males not receiving antidepressant treatment) than those who do not receive benzodiazepines (Neutel \& Patten, 1997). Whether this relationship is causal is difficult to establish.

In a recent literature review Youssef \& Rich (2008) were unable to draw firm conclusions regarding the role of sedatives/hypnotics in suicide prevention although they did note that hypnotic ingestion was reported in a significant proportion of suicides and thus prescribing should be avoided for this reason.

\section{ECT}

The role of ECT in suicide prevention is difficult to evaluate as there have been no randomised control trials for this indication. Early studies suggested that ECT could be useful in reducing suicide. Avery \& Winokur (1978) reported a lower frequency of suicide in those who had received ECT but Flood \& Seager (1968) reported this effect to be limited to women. Bradvik \& Berglund (2006) assessed the relationship between long term treatments of depressive episodes and attempted and completed suicide. They found that significantly fewer suicide attempts were made by those who had received ECT when compared with those who were prescribed antidepressants, but those on antidepressants made less serious suicide attempts (albeit more frequently). In an earlier paper the same authors suggested that psychomotor retardation is a risk factor for suicide post-ECT (Bradvik \& Berglund, 2005) and Sharma (1999) concluded that ECT was effective as an acute treatment but had no longer term benefit. Although these results could simply reflect the severity of disease it may represent a relapse of the depressive episode for which patients were receiving treatment. Some authors have suggested that as many as $50 \%$ of patients may relapse after ECT (Bourgon \& Kellner, 2000).

\section{MEDICATION FOR PERSONALITY DISORDER}

The evolution of psychotherapeutic interventions for those with personality disorders has led to medication playing an increasingly minor role in suicide prevention in this group, reflected in the absence of any recent studies in this area. Flupenthixol given as a monthly $20 \mathrm{mg}$ depot to those with histrionic, borderline or dependent personality disorders with a history of repetitive self-harm was associated with a reduction in selfharm compared with placebo over 6 months (Montgomery \& Montgomery, 1982; Montgomery et al., 1979). This finding has not been replicated. There has been some interest in the role of SSRIs in reducing repetitive self-harm. Verkes et al. (1998) conducted a 1year double blind placebo control trial investigating the effect of paroxetine $40 \mathrm{mg}$ on subsequent suicidal behaviour in patients that had a history of at least 2 previous acts of self-harm who did not meet criteria for a major depressive disorder. The majority had at least one cluster B personality disorder. There was no difference in self-harm repetition rates between patients on paroxetine and those on placebo. However, a post hoc analysis suggested a preventative effect of paroxetine in patients with fewer than five previous acts of self-harm at entry to the trial, and in those who had fewer cluster B symptoms. There was no difference between the placebo and treatment groups with regard to depressive symptoms, anger or hopelessness. There is limited evidence that fluoxetine may assist in reducing aggression in patients with personality disorders (Coccaro \& Kavoussi, 1997).

\section{CONCLUSION}

Psychotropic medication may have a significant role in suicide prevention. This is most striking for lithium and clozapine. Consistent medication concordance appears to have a protective effect with regard to all mood stabilisers. The augmentation of mood stabilisation with antidepressants or antipsychotic in bipolar disorder should be accompanied by an increased awareness of suicide risk in this group. The use of antidepressants in unipolar depression (especially in the under 18s) remains contentious but the balance of evidence appears to favour active treatment with careful monitoring in younger age groups. Caution should be observed when prescribing hypnotic medication as there is little evidence to support the commonly held view that it is helpful in reducing risk during the early stages of antidepressant treatment, and some suggestion that it may increase suicidal behaviour. At present the evidence for the use of medication in personality disorder is inconclusive. 


\section{REFERENCES}

Angst J. \& Clayton P. (1986). Premorbid personality of depressive, bipolar, and schizophrenic patients with special reference to suicidal issues. Comprehensive Psychiatry 27, 511-532.

Avery D. \& Winokur G. (1978). Suicide, attempted suicide, and relapse rates in depression. Archives of General Psychiatry 35, 749-753.

Baldessarini R.J., Tondo L. \& Hennen J. (2003). Lithium treatment and suicide risk in major affective disorders: update and new findings. Journal of Clinical Psychiatry 64, Suppl. 5, 44-52.

Baldessarini R.J., Pompili M. \& Tondo L. (2006). Suicidal risk in antidepressant drug trials. Archives of General Psychiatry 63, 246-248.

Bauer M.S., Wisniewski S.R., Kogan J.N., Marangell L.B., Thase M.E. $\&$ Sachs G. (2006). Brief report: paroxetine in younger and adult individuals at high risk for suicide. Psychopharmacology Bulletin 39, 31-37.

Bourgon L.N. \& Kellner C.H. (2000). Relapse of depression after ECT: a review. Journal of ECT 16, 19-31.

Bradvik L. \& Berglund M. (2005). Suicide in severe depression related to treatment: depressive characteristics and rate of antidepressant overdose. European Archives of Psychiatry and Clinical Neuroscience 255, 245-250.

Bradvik L. \& Berglund M. (2006). Long-term treatment and suicidal behavior in severe depression: ECT and antidepressant pharmacotherapy may have different effects on the occurrence and seriousness of suicide attempts. Depression and Anxiety 23, 34-41.

Bridge J.A., Iyengar S., Salary C.B., Barbe R.P., Birmaher B., Pincus H.A., Ren L. \& Brent D.A. (2007). Clinical response and risk for reported suicidal ideation and suicide attempts in pediatric antidepressant treatment: a meta-analysis of randomized controlled trials. Journal of American Medical Association 297, 1683-1696.

Cavanagh J.T., Carson A.J., Sharpe M. \& Lawrie S.M. (2003). Psychological autopsy studies of suicide: a systematic review. Psychological Medicine 33, 395-405.

Cipriani A., Pretty H., Hawton K. \& Geddes J.R. (2005). Lithium in the prevention of suicidal behavior and all-cause mortality in patients with mood disorders: a systematic review of randomized trials. American Journal of Psychiatry 162, 1805-1819.

Coccaro E.F. \& Kavoussi R.J. (1997). Fluoxetine and impulsive aggressive behavior in personality-disordered subjects. Archives of General Psychiatry 54, 1081-1088.

Fawcett J., Scheftner W.A., Fogg L., Clark D.C., Young M.A., Hedeker D. \& Gibbons R. (1990). Time-related predictors of suicide in major affective disorder. American Journal of Psychiatry 147, 1189-1194.

Fenton W.S., McGlashan T.H., Victor B.J. \& Blyler C.R. (1997). Symptoms, subtype, and suicidality in patients with schizophrenia spectrum disorders. American Journal of Psychiatry 154, 199-204.

Fergusson D., Doucette S., Glass K.C., Shapiro S., Healy D., Hebert P. \& Hutton B. (2005). Association between suicide attempts and selective serotonin reuptake inhibitors: systematic review of randomised controlled trials. British Medical Journal 330, 396.

Flood R.A. \& Seager C.P. (1968). A retrospective examination of psychiatric case records of patients who subsequently committed suicide. British Journal of Psychiatry 114, 443-450.

Geddes J., Burgess S., Hawton K., Jamison K. \& Goodwin G.M. (2004). Long-term lithium therapy for bipolar disorder: systematic review and meta-analysis of randomized controlled trials. American Journal of Psychiatry 161, 217-222.

Gibbons R.D., Brown C.H., Hur K., Marcus S.M., Bhaumik D.K., Erkens J.A., Herings R.M. \& Mann J.J. (2007). Early evidence on the effects of regulators' suicidality warnings on SSRI prescriptions and suicide in children and adolescents. American Journal of Psychiatry 164, 1356-1363.

Goldberg J.F., Allen M.H., Miklowitz D.A., Bowden C.L., Endick C.J., Chessick C.A., Wisniewski S.R., Miyahara S., Sagduyu K., Thase M.E., Calabrese J.R. \& Sachs G.S. (2005). Suicidal ideation and pharmacotherapy among STEP-BD patients. Psychiatric Services 56, 15341540.
Goodwin F.K., Fireman B., Simon G.E., Hunkeler E.M., Lee J. \& Revicki D. (2003). Suicide risk in bipolar disorder during treatment with lithium and divalproex. Journal of the American Medical Association 290, 1467-1473.

Hall R.C. \& Joffe J.R. (1972). Aberrant response to diazepam: a new syndrome. American Journal of Psychiatry 129, 738-742.

Haw C., Hawton K., Houston K. \& Townsend E. (2001). Psychiatric and personality disorders in deliberate self-harm patients. British Journal of Psychiatry 178, 48-54.

Hawton K., Sutton L., Haw C., Sinclair J. \&, Deeks J.J. (2005a). Schizophrenia and suicide: systematic review of risk factors. British Journal of Psychiatry 187, 9-20.

Hawton K., Sutton L., Haw C., Sinclair J. \& Harriss L. (2005b). Suicide and attempted suicide in bipolar disorder: a systematic review of risk factors. Journal of Clinical Psychiatry 66, 693-704.

Jonas J.M. \& Hearron A.E., Jr. (1996). Alprazolam and suicidal ideation: a meta-analysis of controlled trials in the treatment of depression. Journal of Clinical Psychopharmacology 16, 208-211.

Khan A., Khan S.R., Leventhal R.M. \& Brown W.A. (2001). Symptom reduction and suicide risk among patients treated with placebo in antipsychotic clinical trials: an analysis of the food and drug administration database. American Journal of Psychiatry 158, 1449-1454.

Khan A., Khan S., Kolts R. \& Brown W.A., 2003. Suicide rates in clinical trials of SSRIs, other antidepressants, and placebo: analysis of FDA reports. American Journal of Psychiatry 160, 790-792.

Lauterbach E., Felber W., Muller-Oerlinghausen B., Ahrens B., Bronisch T., Meyer T., Kilb B., Lewitzka U., Hawellek B., Quante A., Richter K., Broocks A. \& Hohagen F. (2008). Adjunctive lithium treatment in the prevention of suicidal behaviour in depressive disorders: a randomised, placebo-controlled, 1-year trial. Acta Psychiatrica Scandinavica 118, 469-479.

Leon A.C., Marzuk P.M., Tardiff K. \& Teres, J.J. (2004). Paroxetine, other antidepressants, and youth suicide in New York City: 1993 through 1998. Journal of Clinical Psychiatry 65, 915-918.

Lindenmayer J.P., Czobor P., Alphs L., Nathan A.M., Anand R., Islam Z. \& Chou J.C. (2003). The InterSePT scale for suicidal thinking reliability and validity. Schizophrenia Research 63, 161-170.

Mann J.J., Apter A., Bertolote J., Beautrais A., Currier D., Haas A., Hegerl U., Lonnqvist J., Malone K., Marusic A., Mehlum L., Patton G., Phillips M., Rutz W., Rihmer Z., Schmidtke A., Shaffer D., Silverman M., Takahashi Y., Varnik A., Wasserman D., Yip P. \& Hendin H. (2005). Suicide prevention strategies: a systematic review. Journal of the American Medical Association 294, 2064-2074.

Meltzer H.Y. \& Okayli G. (1995). Reduction of suicidality during clozapine treatment of neuroleptic-resistant schizophrenia: impact on risk-benefit assessment. American Journal of Psychiatry 152, 183-190.

Meltzer H.Y., Alphs L., Green A.I., Altamura A.C., Anand R., Bertoldi A., Bourgeois M., Chouinard G., Islam M.Z., Kane J., Krishnan R., Lindenmayer J.P. \& Potkin, S. (2003). Clozapine treatment for suicidality in schizophrenia: International Suicide Prevention Trial (InterSePT). Archives of General Psychiatry 60, 82-91.

Moller H.J. (2006). Evidence for beneficial effects of antidepressants on suicidality in depressive patients: a systematic review. European Archives of Psychiatry and Clinical Neuroscience 256, 329-343.

Montgomery S.A. \& Montgomery D. (1982). Pharmacological prevention of suicidal behaviour. Journal of Affective Disorders 4, 291-298.

Montgomery S.A., Montgomery D.B., Rani S.J., Roy P.H., Shaw P.J. \& McAuley R. (1979). Maintenance therapy in repeat suicide behaviour - A Placebo controlled trial. In Proceedings of the 10th International Congress of Suicide Prevention and Crisis Intervention, pp. 227-229.

Muller-Oerlinghausen B., Muser-Causemann B. \& Volk J. (1992). Suicides and parasuicides in a high-risk patient group on and off lithium long-term medication. Journal of Affective Disorders 25, 261-269.

Neutel C.I. \& Patten S.B. (1997). Risk of suicide attempts after benzodiazepine and/or antidepressant use. Annals of Epidemiology 7, 568574 .

Epidemiologia e Psichiatria Sociale, 18, 3, 2009 
Palmer B.A., Pankratz V.S. \& Bostwick J.M. (2005). The lifetime risk of suicide in schizophrenia: a reexamination. Archives of General Psychiatry 62, 247-253.

Rihmer Z. \& Akiskal H. (2006). Do antidepressants t(h)reat(en) depressives? Toward a clinically judicious formulation of the antidepressant-suicidality FDA advisory in light of declining national suicide statistics from many countries. Journal of Affective Disorders 94, 3-13.

Roy A. (1982). Suicide in chronic schizophrenia. British Journal of Psychiatry 141, 171-177.

Ryan H.F., Merrill F.B., Scott G.E., Krebs R. \& Thompson B.L. (1968). Increase in suicidal thoughts and tendencies. Association with diazepam therapy. Journal of the American Medical Association 203, 1137-1139.

Sernyak M.J., Desai R., Stolar M. \& Rosenheck R. (2001). Impact of clozapine on completed suicide. American Journal of Psychiatry $158,931-937$.

Sharma V. (1999). Retrospective controlled study of inpatient ECT: does it prevent suicide? Journal of Affective Disorders 56, 183-187.

Shi L., Thiebaud P. \& McCombs J.S. (2004). The impact of unrecognized bipolar disorders for patients treated for depression with antidepressants in the fee-for-services California Medicaid (Medi-Cal) program. Journal of Affective Disorders 82, 373-383.

Simon G.E., Savarino J., Operskalski B. \& Wang P.S. (2006). Suicide risk during antidepressant treatment. American Journal of Psychiatry 163, 41-47.

Smith E.G., Sondergard L., Lopez A.G., Andersen P.K. \& Kessing L.V. (2009). Association between consistent purchase of anticonvulsants or lithium and suicide risk: A longitudinal cohort study from Denmark, 1995-2001. Journal of Affective Disorders Feb 23 [Epub ahead of print].

Sondergard L., Lopez A.G. Andersen P.K. \& Kessing L.V. (2008). Mood-stabilizing pharmacological treatment in bipolar disorders and risk of suicide. Bipolar Disorders 10, 87-94.

Tondo L. \& Baldessarini R.J. (2000). Reduced suicide risk during lithium maintenance treatment. Journal of Clinical Psychiatry 61, Suppl. 9, 97-104.

Tran P.V., Hamilton S.H., Kuntz A.J., Potvin J.H., Andersen S.W., Beasley C., Jr. \& Tollefson G.D. (1997). Double-blind comparison of olanzapine versus risperidone in the treatment of schizophrenia and other psychotic disorders. Journal of Clinical Psychopharmacology 17, 407-418.
Valuck R.J., Libby A.M., Sills M.R., Giese A.A. \& Allen R.R. (2004). Antidepressant treatment and risk of suicide attempt by adolescents with major depressive disorder: a propensity-adjusted retrospective cohort study. CNS Drugs 18, 1119-1132.

Verkes R.J., Van der Mast R.C., Hengeveld M.W., Tuyl J.P., Zwinderman A.H. \& Van Kempen G.M. (1998). Reduction by paroxetine of suicidal behavior in patients with repeated suicide attempts but not major depression. American Journal of Psychiatry $155,543-547$.

Walker A.M., Lanza L.L., Arellano F. \& Rothman K.J. (1997). Mortality in current and former users of clozapine. Epidemiology 8, 671-677.

Ward A., Ishak K., Proskorovsky I. \& Caro J. (2006). Compliance with refilling prescriptions for atypical antipsychotic agents and its association with the risks for hospitalization, suicide, and death in patients with schizophrenia in Quebec and Saskatchewan: a retrospective database study. Clinical Therapy 28, 1912-1921.

Wheeler B.W., Gunnell D., Metcalfe C., Stephens P. \& Martin R.M. (2008). The population impact on incidence of suicide and non-fatal self harm of regulatory action against the use of selective serotonin reuptake inhibitors in under 18s in the United Kingdom: ecological study. British Medical Journal 336, 542-545.

Wickham E.A. \& Reed J.V. (1987). Lithium for the control of aggressive and self-mutilating behaviour. International Clinical Psychopharmacology 2, 181-190.

Winokur G. \& Tsuang M. (1975). The Iowa 500: suicide in mania, depression, and schizophrenia. American Journal of Psychiatry 132, 650-651.

Yerevanian B.I., Koek R.J. \& Mintz J. (2003). Lithium, anticonvulsants and suicidal behavior in bipolar disorder. Journal of Affective Disorders 73, 223-228.

Yerevanian B.I., Koek R.J. \& Mintz J. (2007a). Bipolar pharmacotherapy and suicidal behavior: Part 3: Impact of antipsychotics. Journal of Affective Disorders 103, 23-28.

Yerevanian B.I., Koek R.J., Mintz J. \& Akiskal H.S. (2007b). Bipolar pharmacotherapy and suicidal behavior: Part 2. The impact of antidepressants. Journal of Affective Disorders 103, 13-21.

Youssef N.A. \& Rich C.L. (2008). Does acute treatment with sedatives/hypnotics for anxiety in depressed patients affect suicide risk? A literature review. Annals of Clinical Psychiatry 20, 157169. 